Original Paper.

\title{
Sensitive Mercury Speciation Analysis in Water by High Performance Liquid Chromatography-Atomic Fluorescence Spectrometry Coupling with Solid Phase Extraction
}

Dongyang Chen, ${ }^{\mathrm{a}}$ Lan Lu, ${ }^{\mathrm{a}}$ Hao Zhang, ${ }^{\mathrm{a}}$ Bing Lu. ${ }^{\mathrm{a}}$ Jiali Feng, ${ }^{\dagger}$ a Dong Zeng a

${ }^{a}$ Hunan Provincial Center for Disease Control and Prevention, Changsha 410005, China.

\begin{abstract}
An efficient method based on high performance liquid chromatography coupled with atomic fluorescence spectrometry (HPLC-AFS) was successfully developed for the simultaneous determination of four mercury species including $\mathrm{Hg}^{2+}$, methylmercury ( $\mathrm{MeHg})$, ethylmercury $(\mathrm{EtHg})$, and phenylmercury $(\mathrm{PhHg})$ in water. Samples were enriched and cleaned up with solid phase extraction (SPE) pretreatment using thiol cartridge, some key parameters including selection of SPE cartridge, eluent type, eluent volume, and the interference factors were systematically investigated. The chromatographic separation was achieved on $\mathrm{C}_{18}$ column using a mobile phase consisting of methanol and $60 \mathrm{mmol} \mathrm{L}^{-1}$ ammonium acetate with $10 \mathrm{mmol} \mathrm{L}^{-1}$ L-cysteine by gradient elution. Under the optimized conditions, good linearity $(\mathrm{r} \geq$ 0.9991 ) was observed between $0.20 \mu \mathrm{g} \mathrm{L}^{-1}$ to $10.0 \mu \mathrm{g} \mathrm{\textrm {L } ^ { - 1 }}$. The limits of detection were
\end{abstract}

† Corresponding author, Email address: goodlucklhk@163.com 
in the ranges of $0.001 \mu \mathrm{g} \mathrm{L^{-1 }}-0.002 \mu \mathrm{g} \mathrm{L}^{-1}$, high recoveries $(87.2 \%$ to $111 \%$ ) and good reproducibility $(1.1 \%-6.5 \%)$ were obtained. Such method is sensitive, selective and accurate, which can be applied to the quantification of mercury species in water samples. 


\section{Introduction}

Mercury recognized as one of the most toxic elements presents great harmful effects on human health. ${ }^{1}$ However, total mercury is inadequate to present its eco-toxicity, whose toxicity and metabolic behaviors depend much on its chemical form. Organomercury displaying more toxic than inorganic ones, have gained considerable attention because of their lipophilicity and bioaccumulation characters. ${ }^{2}$ The common mercury species found in water are inorganic mercury $\left(\mathrm{Hg}^{2+}\right)$, alkylmercury (methylmercury (MeHg) and ethylmercury (EtHg)), and phenylmercury (PhHg) (Fig 1). These mercury species in water particularly attract great concerns because they may transport to soil, plant, fish, and finally to human through food chain. ${ }^{3}$ Therefore, it is significant to develop sensitive and accurate analytical techniques for such mercury species in water.

\section{Fig 1}

There have been many efforts devoted to detect mercury species, such as gas chromatography (GC), ${ }^{4}$ high performance liquid chromatography (HPLC), ${ }^{5}$ gas chromatography- mass spectrometry (GC-MS), ${ }^{6}$ and capillary electrophoresis (CE). ${ }^{7}$ However, GC and GC-MS technologies require derivatization, which is commonly considered time-consuming and laborious, while HPLC and CE present low sensitivity. Nowadays, the common approach for the mercury species detection is to hyphenate a sensitive element-selective detector to a powerful separation technology, ${ }^{8-11}$ and high performance liquid chromatography coupled with atomic fluorescence spectrometry (HPLC-AFS) and high performance liquid chromatography-inductively coupled plasma mass spectrometry(HPLC-ICP-MS) are the mostly applied analytical techniques for

such purpose. ${ }^{12-14}$ HPLC-ICP-MS displays excellent sensitivity but the operational cost 
is high, and the instrument is too expensive to widely use in basic laboratories. In contrast, HPLC-AFS is preferable for qualitative and quantitative determination with excellent precision, accuracy and lower cost, which is much more practical and economical in detecting mercury species. In additionally, a further preconcentration procedure is essential for mercury species determination because of their trace levels in water samples such as liquid-liquid extraction (LLE), ${ }^{15}$ liquid-liquid-liquid microextraction (LLLME), ${ }^{16}$ distillation, ${ }^{17}$ and solid phase extraction (SPE). ${ }^{18,19}$ Among these techniques, SPE displays attractive advantages for its flexibility, high retention capacity, ease of automation and minimal consumption of organic solvents. Shirkhanloo and coworker prepared carboxyl-functionalized nanoporous graphene as solid phase sorbent for the speciation analysis of $\mathrm{Hg}^{2+}$ and $\mathrm{MeHg}$, the method achieved high recoveries and good preconcentration factor. ${ }^{20}$ Liu reported a simple SPE pretreatment using the commercially available $\mathrm{C}_{18}$ cartridge to trap $\mathrm{Hg}^{2+}$, $\mathrm{MeHg}$, and $\mathrm{EtHg} .{ }^{21}$ But this cartridge required further pre-functionalization with sulphur compounds. In recent years, some novel adsorbents also were introduced to enrich low-content mercury species in water and obtained satisfactory enriching effects. ${ }^{22,24}$ However, these home-made SPE cartridges could not tolerate large volume sample. Selecting a suitable SPE cartridge remains essential for sensitive mercury speciation in water. The development of sensitive and reliable method for the mercury speciation analysis in water is still significant and timely considering the high toxicity of these compounds.

The objective of this study is to develop an efficient method based on SPE coupling with HPLC-AFS for the simultaneous determination of four mercury species in water samples. We aim to seek a simple, efficient SPE procedure, which possesses high adsorption capacity and can tolerate large-volume water sample. The key parameters that affected sample preparation and determination were optimized through a series of 
tests. And the sensitivity and accuracy of the method were also evaluated.

\section{Experimental}

\section{Reagents and chemicals}

$\mathrm{Hg}^{2+}$ standard stock solution $\left(1000 \mathrm{mg} \mathrm{L}^{-1}\right)$ was purchased from national research center for standard materials (China). Methylmercury chloride, ethylmercury chloride, and phenylmercury chloride were obtained from Dr. Ehrenstorfer GmbH (Germany). HPLC grade methanol was obtained from Merck (Germany). Guaranteed reagent hydrochloric acid, $\mathrm{KBH}_{4}$, L-cysteine, thiourea and ammonium acetate were provided by Sinopharm chemical reagent Co., Ltd. (China). Lobster hepatopancreas certified reference material (TORT-3) was purchased from national research council Canada. The stock solutions of organomercury were prepared by dissolving appropriate amounts of standards in methanol, and these solutions were appropriately diluted with $0.4 \%$ hydrochloric acid to prepare the standard working solutions. Water used was purified (18 $\mathrm{M} \Omega \cdot \mathrm{cm}$ quality) by a Milli-Q system (Millipore, Bedford, USA).

Thiol cartridge (50 mg, $3 \mathrm{~mL}$ ) was purchased from ANPEL laboratory technologies (China), Oasis HLB (60 mg, $3 \mathrm{~mL}), \mathrm{C}_{18}(500 \mathrm{mg}, 6 \mathrm{~mL})$, MAX (150 mg, $\left.6 \mathrm{~mL}\right)$, activated carbon (400 mg, $0.7 \mathrm{~mL}$ ) were provided by Waters (Milford, USA). Filters membrane $0.45 \mu \mathrm{m}$ of polyether sulfone were purchased from Xiboshi (Tientsin, China). To avoid $\mathrm{Hg}$ residual, all the glass and plastic vessels soaked in $5 \% \mathrm{HNO}_{3}$ overnight, and then cleaned with deionized water.

\section{Detection conditions}

The HPLC-AFS system (SA-50) was offered by Beijing titan instruments Co., Ltd. (China). Chromatographic separation was achieved with a Diamonsil $\mathrm{C}_{18}$ column (4.6 $\mathrm{mm} \times 250 \mathrm{~mm}, 5 \mu \mathrm{m}$, Dikma, China). The mobile phase system was consisted of 
solution A (methanol) and $\mathrm{B}\left(60 \mathrm{mmol} \mathrm{L}^{-1}\right.$ ammonium acetate with $10 \mathrm{mmol} \mathrm{L}{ }^{-1}$ L-cysteine). A gradient program was used for elution: 0-6 min, $2 \%$ A, $6-11$ min, $2 \%$ $60 \%$ A, 11-15 min, $60 \%$ A, $15-16 \min , 60-2 \%$ A, 16-20 min, $2 \%$ A. The column temperature was $25^{\circ} \mathrm{C}$. The flow rate was set as $1.0 \mathrm{~mL} \mathrm{~min}{ }^{-1}$, The sample volume injected was $100 \mu \mathrm{L}$. AFS conditions were as follow: lamp wavelength: $253.7 \mathrm{~nm}$; lamp current: $40 \mathrm{~mA}$; carrier gas: $400 \mathrm{~mL} \mathrm{~min}{ }^{-1}$; PMT voltage: $300 \mathrm{~V}$; auxiliary gas: $500 \mathrm{~mL}$ $\min ^{-1}$; carrier solution: $7 \% \mathrm{HCl}$; reducing agent: $0.50 \% \mathrm{KBH}_{4}$ in $0.50 \% \mathrm{KOH}$ solution.

\section{Sample preparation}

Water samples were preserved by adding $4 \mathrm{~mL}$ of concentrated hydrochloric acid (12 mol $\mathrm{L}^{-1}$ ) per liter. Prior to analysis, water sample was filtered through a $0.45 \mu \mathrm{m}$ membrane filter. A $200 \mathrm{~mL}$ volume of the filtered water was passed through a thiol cartridge, which was preconditioned with $5 \mathrm{~mL} 0.4 \%$ hydrochloric acid. After the extraction cartridge was washed with $5 \mathrm{~mL}$ of purified water, it was dried by nitrogen for $3 \mathrm{~min}$. The target compounds collected on the cartridge were eluted with $4 \mathrm{~mL}$ of 7 mol L ${ }^{-1} \mathrm{HCl}$. The eluate was adjusted to $\mathrm{pH} 4 \sim \mathrm{pH} 7$ using ammonia solution, and added initial mobile phase solution $\left(60 \mathrm{mmol} \mathrm{L} \mathrm{L}^{-1}\right.$ ammonium acetate containing with $2 \%$ methanol and $10 \mathrm{mmol} \mathrm{L}{ }^{-1} \mathrm{~L}$-cysteine) to make $5.0 \mathrm{~mL}$. The final solution mixed well by a vortex shaker, then was filtered through a $0.45 \mu \mathrm{m}$ polyether sulfone membrane filter and transferred into amber glass vials for HPLC-AFS analysis. Blank sample was operated in the same conditions.

\section{Results and Discussion}

\section{Optimization of detection conditions}

Four mercury species were separated on a reversed-phased $\mathrm{C}_{18}$ column with a mobile 
phase of methanol and ammonium acetate solution. It took at least $40 \mathrm{~min}$ under isocratic elution program resulting obvious tailing of the $\mathrm{PhHg}$ chromatographic peak. Therefore, gradient elution mode was adopted. In order to enhance the elution ability of the mobile phase and improve the peak symmetry, sulfur-containing chelating agent was added to the mobile phase to form the corresponding $\mathrm{Hg}$ complex. ${ }^{25,26}$ L-cysteine, 2-mercaptoethanol, and diethyldithiocarbamate were investigated. The results showed that with the addition of L-cysteine or 2-mercaptoethanol were beneficial to the peak symmetry, four mercury species achieved absolute separation. In view of the toxicity and terrible smell of 2-mercaptoethanol, L-cysteine was selected as the complexing agent added into mobile phase. Moreover, the effect of the L-cysteine concentration in the range of $2 \mathrm{mmol} \mathrm{L}^{-1}-20 \mathrm{mmol} \mathrm{L} \mathrm{L}^{-1}$ on the separation performance was studied as well. The chromatographic peak symmetries was significantly improved as the L-cysteine concentration up to $10 \mathrm{mmol} \mathrm{L}^{-1}$ leading to remarkable improvement of the sensitivities. Hence, $10 \mathrm{mmol} \mathrm{L}^{-1}$ of L-cysteine was chosen as the mobile phase additive for the subsequent experiments (Fig 2). The effect of mobile phase $\mathrm{pH}$ on separation was also investigated by changing the $\mathrm{pH}$ from 2.0 to 7.0. No obvious change was found in the chromatograms. Therefore, the mobile phase solution was prepared without $\mathrm{pH}$ adjustment.

The AFS conditions were further optimized. In generally, the carrier gas used in AFS was argon, which used to bring element mercury into the atomizer. Herein, the flow rate of carrier gas was optimized in the range of $200 \mathrm{~mL} \mathrm{~min}^{-1}$ to $700 \mathrm{~mL} \mathrm{~min}{ }^{-1}$. The most sensitive results were obtained with the gas flow rate at $400 \mathrm{~mL} \mathrm{~min}^{-1}$, which could be ascribed to the facts that lower carrier flow rate could not bring element mercury into atomizer efficiently, while excessive flow rate would dilute the concentrations of element mercury in atomizer. Thus, the flow rate of carrier gas was set at $400 \mathrm{~mL} \mathrm{~min}^{-1}$. 
Appropriate amounts of $\mathrm{KBH}_{4}$ and hydrochloric acid were significant for the sensitivity of AFS detector. The effects of the hydrochloric acid concentration ranging from $5 \%$ to $12 \%$ and $\mathrm{KBH}_{4}$ concentration ranging from $0.20 \%$ to $1.0 \%$ on the sensitivities were systematically investigated. The signal intensities of mercury species increased at first and then decreased with the $\mathrm{KBH}_{4}$ concentration increased, such result could be explained by the excessive hydrogen would dilute the concentrations of element mercury in atomizer. The highest mercury species atomic fluorescence signals were obtained with $7 \%$ hydrochloric acid and $0.50 \% \mathrm{KBH}_{4}$.

Fig 2

\section{Optimization of SPE procedure}

An appropriate cartridge is of major importance for the SPE method. HLB cartridge, $\mathrm{C}_{18}$ cartridge, MAX cartridge, thiol cartridge, and activated carbon cartridge were selected for the SPE pretreatment. According to the previous report, ${ }^{27}$ HLB and $\mathrm{C}_{18}$ cartridges were modified with sodium diethyldithiocarbamate (DDT) to enhance the mercury capture ability. $3 \mathrm{~mL}$ modifier $(0.05 \%$ DDT) and $5 \mathrm{~mL}$ water were further added at preconditioned step, and the eluent used was $10 \mathrm{~mL}$ acetonitrile and evaporated near dryness under a stream of nitrogen, and then redissolved with $1 \mathrm{~mL} 0.4 \%$ hydrochloric acid. $0.05 \mathrm{~mol} \mathrm{~L}^{-1}$ spiked tap water samples were used for the optimization of SPE procedure. The values of the detection results were consisted of average value \pm standard deviation (SD), which were obtained by three parallel experiments.

Fig 3 showed that thiol cartridge exhibited the highest recoveries ranging from $84.6 \%$ to $108 \%$, followed by $\mathrm{C}_{18}$ and HLB cartridges, the latter recoveries were $46.2 \%$ $83.1 \%$ and $33.1 \%-72.8 \%$, respectively. While activated carbon cartridge and MAX cartridge displayed unsatisfactory performance. The functional group named sulfur donor atom in thiol adsorbent possessed a high complexing capability with $\mathrm{Hg}$ resulting 
high recoveries, ${ }^{28,29}$ and such cartridge did not need further functionalization. DDT-functionalized $\mathrm{C}_{18}$ and HLB could efficiently preserve mercury species for small volume samples, while the recoveries deceased seriously as the loading volume exceeded $50 \mathrm{~mL}$. The activated carbon exhibited excellent retention capacity for the mercury species as well. However, it is unable to elute the mercury species efficiently from the cartridge with various solvents resulting low recoveries. Surprisingly, the retention time of organomercury migrated seriously after eluting from MAX cartridge. Therefore, the thiol cartridge was optimum. On the other hand, the recoveries of EtHg and $\mathrm{PhHg}$ decreased significantly as the sample volume was higher than $200 \mathrm{~mL}$. The recoveries of $\mathrm{EtHg}$ and $\mathrm{PhHg}$ were respectively $98.8 \%$ and $92.1 \%$ at $200 \mathrm{~mL}$ loading volume, while these values dropped to $86.7 \%$ and $76.4 \%$ for $220 \mathrm{~mL}$ loading sample, then dropped to $79.1 \%$ and $62.3 \%$ at $240 \mathrm{~mL}$ loading volume. Such results could be ascribed to a possible breakthrough of the analytes on the cartridge with the increase of the loading sample.

\section{Fig 3}

Appropriate elution solvent plays an important role in the SPE procedure. $\mathrm{HCl}$ was an efficient eluent for the sulfhydryl cotton fiber absorbent. ${ }^{30}$ Thus, different concentrations of $\mathrm{HCl}$ were compared for their elution efficiencies (Fig 4a). Along with the raise of $\mathrm{HCl}$ concentration, the recoveries of the analytes increased. The recoveries of $\mathrm{MeHg}$ and $\mathrm{EtHg}$ trended to plateau as the $\mathrm{HCl}$ concentration was higher than $4 \mathrm{~mol}$ $\mathrm{L}^{-1}$, the $\mathrm{PhHg}$ recovery held steady from $7 \mathrm{~mol} \mathrm{~L}^{-1} \mathrm{HCl}$ up, and the recovery of $\mathrm{Hg}^{2+}$ had not peaked under the investigated concentration but the value had exceed $85 \%$ at 7 mol $\mathrm{L}^{-1} \mathrm{HCl}$. Thiourea, L-cysteine, and mercaptoethanol were considered to be beneficial for mercury elution. ${ }^{31}$ By comparing the recoveries obtained with the complexing agents L-cysteine and thiourea and considering the high toxicity of mercaptoethanol, thiourea 
was chosen to add into $5 \mathrm{~mol} \mathrm{~L}{ }^{-1} \mathrm{HCl}$ as elution solvent, and the effect of different concentration of thiourea on the mercury species recoveries was further investigated (Fig 4b). The recoveries of $\mathrm{Hg}^{2+}$ and $\mathrm{PhHg}$ aggrandized significantly with the thiourea concentration increased. However, we also noticed that the recovery of $\mathrm{PhHg}$ gradually decreased as the thiourea concentration was higher than $0.025 \%$, while that of $\mathrm{Hg}^{2+}$ was abnormal high (> $120 \%)$. It was hypothesized that excess thiourea might weaken or replace the $\mathrm{C}-\mathrm{Hg}$ bond of organomercury by chelating with them, which would generate new complexes consequently peaked at divalent mercury retention time. The same phenomenon occurred in the solitary $\mathrm{PhHg}$ sample solution, which well supported the assumption. From the bond energy perspective, phenyl is electrondrawing group, while the alkyl is electron-donating group. The $\mathrm{C}$ - $\mathrm{Hg}$ binding energy of alkylmercury is larger than that of $\mathrm{PhHg}$. Consequently, the trend of forming complexes was $\mathrm{PhHg}>$ alkylmercury. The similar phenomenons were ever reported in some literatures. ${ }^{32}$ Considering the converting yield might depend on the ratio of the $\mathrm{PhHg}$ : thiourea concentration, optimum thiourea concentration may be unfixed at $0.025 \%$ in the case of utilization for an unknown sample. $7 \mathrm{~mol} \mathrm{~L}^{-1} \mathrm{HCl}$ was adopted as eluent.

\section{Fig 4}

\section{Fig 5}

In additionally, the volume of elution solvent is another important factor for SPE method. The effect of elution volume ranging from $1.0 \mathrm{~mL}$ to $9.0 \mathrm{~mL}$ on the recoveries was investigated. As shown in Fig 5, the recoveries of mercury species increased with the increasing eluent volume. The recoveries of organomercury $(\mathrm{MeHg}, \mathrm{EtHg}$, and $\mathrm{PhHg}$ ) reached to a stable level by using $3 \mathrm{~mL}$ eluent. $\mathrm{Hg}^{2+}$ was difficult to elute because the force of $\mathrm{Hg}^{2+}$-thiol chelate was stronger than that of organomercury-thiol chelate. Therefore, $4.0 \mathrm{~mL}$ eluent was selected as optimum. 


\section{Interferences}

Anti-interference ability was significant for the proposed method. The commonly cations $\left(\mathrm{K}^{+}, \mathrm{Na}^{+}, \mathrm{Mg}^{2+}, \mathrm{Ca}^{2+)}\right.$, anions $\left(\mathrm{SO}^{2-}, \mathrm{NO}_{3}^{-}\right)$and some possible pesticide residues in water could not be retained in thiol cartridge. Thus, the major interferences were the coadsorption transition metal ions. The effects of some typical coexisting ions (e.g., $\mathrm{Pb}^{2+}, \mathrm{Ni}^{2+}, \mathrm{Zn}^{2+}, \mathrm{Cd}^{2+}$, and $\mathrm{Cu}^{2+}$ ) on the detection performance were investigated. The ratios of interference for a $\pm 10 \%$ signal change relative to the $0.050 \mu \mathrm{g} \mathrm{L}^{-1}$ analytes were as follows: 1000 -fold for $\mathrm{Ni}^{2+}, \mathrm{Cu}^{2+}, \mathrm{Zn}^{2+}$ and 2000 -fold for $\mathrm{Pb}^{3+}$ and $\mathrm{Cd}^{2+}$. It was important to point out $\mathrm{Cl}^{-}$ion concentration was proved to be a critical factor in mercury species detection, the extraction efficiency decreased when the $\mathrm{Cl}^{-}$ion concentration was higher than $0.54 \mathrm{~mol} \mathrm{~L}{ }^{-1}{ }^{33}$ Therefore, the hyperhaline water samples should be diluted before loading on SPE cartridge.

\section{Method performance}

To check the performance of the proposed method, parameters such as limit of detection (LOD), linearity range, and correlation coefficients were investigated (Table 1). Linearity was studied by analyzing the mixed standard solution at six concentrations ranging from $0.20 \mu \mathrm{g} \mathrm{L}^{-1}$ to $10.0 \mu \mathrm{g} \mathrm{L}^{-1}$ according to the values of the linear correlation coefficients for the calibration curves, and good correlations $(r \geq 0.9991)$ were obtained. LOD was calculated as the amount of analyte that produced a signal to noise ratio of $3: 1$, it was worth noting that blank should be deducted simultaneously. The LOD values were in the range of $0.05 \mu \mathrm{g} \mathrm{L}^{-1}-0.1 \mu \mathrm{g} \mathrm{L}^{-1}$, and the method detection limits were 0.001 $\mu \mathrm{g} \mathrm{L}^{-1}-0.002 \mu \mathrm{g} \mathrm{L}^{-1}$ according to the pretreatment procedure. Such values not only were lower than them of previous reports with similar method $\left(0.002 \mu \mathrm{g} \mathrm{L^{-1 }}-0.01 \mu \mathrm{g}\right.$ $\left.\mathrm{L}^{-1}\right),{ }^{28,34,35}$ but also could be comparable to some HPLC-ICP-MS methods. ${ }^{36,37}$

To evaluate the recovery and precision of the method, six replicates at three different 
spiking levels in various water samples were analyzed (Table 2). The results showed that the recoveries ranged from $87.2 \%$ to $111 \%$, the recoveries of $\mathrm{PhHg}$ were relatively low, which could be ascribed to the biding force between $\mathrm{PhHg}$ and sulfydryl was lowest among the four mercury species, tiny amount of the analyte passed the thiol sorbent. Such results still conformed to the quality control of laboratory with the values were approximately $90 \%$. The relative standard deviations (RSDs) were in the range of $1.1 \%-6.5 \%$. These results demonstrated that the recoveries and precision of the method were satisfied with criterion on quality control of laboratories for chemical testing of water.

\section{Table 1}

\section{Table 2}

\section{Method validation and analysis of samples}

The accuracy of such method was further evaluated by comparing the determination results of two spiked samples and a river water sample with the proposed method and HPLC-ICP-MS method via statistical $T$-test (Table 3). The results showed that a good agreement was found between those two sets of data, which were not significantly different at $95 \%$ confidence $(p<0.05)$. Furthermore, the sum of the mercury species determined using the developed method coincided well with the total mercury content obtained using ICP-MS method. ${ }^{38}$

Subsequently, the obtained method was applied to the analysis of the extracting solution of the reference material NRC TORT-3 (lobster hepatopancreas), which was treated by national standard method, ${ }^{39}$ and the extracting solution was diluted to $200 \mathrm{~mL}$ with $0.4 \%$ hydrochloric acid. The detection results were composed of average value \pm SD obtained by three parallel experiments. The values for $\mathrm{Hg}^{2+}$ and $\mathrm{MeHg}$ were $0.139 \pm 0.022 \mathrm{mg} \mathrm{kg}^{-1}$ and $0.118 \pm 0.015 \mathrm{mg} \mathrm{kg}^{-1}$, respectively, while the certified values 
were $0.155 \pm 0.010 \mathrm{mg} \mathrm{kg}^{-1}$ and $0.137 \pm 0.012 \mathrm{mg} \mathrm{kg}^{-1}$. These results mentioned above proved that the method was acceptable with good accuracy.

\section{Table 3}

Eight water samples including river water, lake water, and tap water were analysed using the proposed method. $\mathrm{Hg}^{2+}$ were found in three river water samples with concentration ranging from 0.43 to $0.79 \mu \mathrm{g} \mathrm{L} \mathrm{L}^{-1}$, while organomercury species were not detected in all water samples.

\section{Conclusions}

An efficient, sensitive and low cost method based on HPLC-AFS coupling with SPE pretreatment for the simultaneous detection of four mercury species in water has been successfully developed. The key factors including detection conditions and SPE parameters were optimized thoroughly. Such method present a good repeatability and high accuracy with satisfactory detection limits, the recoveries ranged from $87.2 \%$ to $111 \%$, RSDs were lower than $6.5 \%$. The proposed method could be applicable to the determination of four mercury species in water samples.

\section{Conflicts of interest}

There are no conflicts to declare

\section{Acknowledgements}

This work was supported by the national institute of environmental health, Chinese center for disease control and prevention (No. 2018shuifa25) and the research project of Hunan provincial health commission (No. B20140134, B20160073 and No. C20190035). 


\section{References}

1. M. Ramos-Osuna, C. Patino-Mejia, J. Ruelas-Inzunza, and O. Escobar-Sanchez, Eviron Monit Assess., 2020, 192, 354.

2. A. Thongsaw, R. SananmuangY. Udnan, G. M. Ross, and W. C. Chaiyasith, Spectrochim. Acta B., 2019, 152, 102.

3. J. Kotnik, M. Horvat, N. Ogrinc, V. Fajon, D. Zagar, D. Cossa, F. Sprovieri, and N. Pirrone, Mar. Pollut. Bull., 2015, 96, 136.

4. Y. Yang, Q. Tan, Y. Lin, Y. F. Tian, L. Wu, X. D. Hou, and C. B. Zheng, Anal Chem., 2018, 90, 11996.

5. Y. Y. Yuan, Y. L. Wu, H. Y. Wang, Y. Y. Tong, X. Y. Sheng, Y. Sun, X. Q. Zhou, and Q. X. Zhou, J. Hazard Mater., 2020, 386, 121658.

6. K. Shigeta, H. Tao, K. Nakagawa, T. Kondo, and T. Nakazato, Anal Sci., 2018, $34,227$.

7. S. L. Wang, X. Y. Song, J. D. Hu, R. Zhang, L. H. Men, M. M. Wei, T. Xie, and J. Cao, Food Chem., 2019, 281, 41.

8. S. Queipo-Abad, C. Lagane, and D. Point, J. Chromatogr. A., 2020, 1617, 460821.

9. Y. Q. Chen, X. Cheng, F. Mo, L. M. Huang, Z. J. Wu, Y. N. Wu, L. J. Xu, and F. F. Fu, Electrophoresis., 2016, 37, 1055.

10. V. Vacchina, F. Seby, R. Chekri, J. Verdeil, J. Dumont, M. Hulin, V. Sirot, J. L. Volatier, R. Serreau, A. Rousseau, T. Simon, and T. Guerin, Talanta., 2017, 167, 404.

11. G. Leng, G. Liu, Y. Chen, H. Yin, and D. Dan, J. Sep. Sci., 2015, 38, 2684.

12. A. A. Krata, and E. Vassileva, Talanta., 2020, 217, 121113. 
13. Y. F. He, M. He, K. Nan, R. K. Cao, B. B. Chen, and B. Hu, J. Chromatogr. A., 2019, 1595, 19.

14. X. L. Zhang, D. L. Ji, Y, Zhang, Y. Lu, J. H. Fu, and Z. H. Wang, J. Anal Atom Spectrom., 2020, 35, 693.

15. H. Pietilä, P. Perämäki, J. Pillspanen, M. Starr, T. Nieminen, M. Kantola, and L. Ukonmaanabho, Chemosphere., 2015, 124, 47.

16. A. A. Gouda, A. M. Alshehri, R. E. Sheikh, W. S . Hassan, S. H. Ibrahim, Microchem J., 2020, 157, 105108.

17. J. R. Miranda-Andrades, S. Khan, M. J. Pedrozo-penafiel, K. D. C. B. Alexandre, R. M. Maciel, R. E. Jr, M. L. B. Tristao, and R. Q. Aucelio, Spectrochim. Acta B., 2019, 158, 105641.

18. B. Duval, A. Gredilla, S. F. O. Vallejuelo, E. Tessier, D. Amouroux, and A. D. Diego, Microchem. J., 2020, 154, 104549.

19. W. J. Jiang, X. Jin, X. H. Yu, W. H. Wu, L. J. Xu, and F. F. Fu, J. Chromatogr. A., 2017, 1496, 167.

20. H. Shirkhanloo, A. Khaligh, H. Z. Mousavi, and A. Rashidi, Microchem. J., 2017, 127, 245.

21. Y. G. Yin, M. Chen, J. F. Peng, J. F. Liu, and G. B. Jiang, Talanta., 2010, 81, 1788

22. A. I. C. Ricardo, A. Sanchez-cachero, M. Jimenez-moreno, F. J. G. Bernardo, and R. C. R. Martin-doimeadios, and A. Rios, Talanta., 2018, 179, 442.

23. M. T. Shi, X. A. Yuan, and W. B. Zhang, Anal. Chim. Acta., 2019, 1074, 33.

24. Z. Es'haghi, G. R. Bardajee, and S. Azimi, Microchem. J., 2016, 127, 170.

25. S. X. Zhang, H. Luo, Y. Y. Zhang, X. Y. Li, J. S. Liu, Q. Xu, and Z. H. Wang, Microchem J., 2016, 126, 25. 
26. S. L. Wang, X. Y. Song, J. D. Hu, R. Zhang, L. H. Men, M. M. Wei, T. Xie, and J. Cao, Food. Chem., 2019, 281, 41.

27. Q. X. Zhou, A. Xing, and K. F. Zhao, J. Chromatogr. A., 2014, 1360, 76.

28. Y. L. Zhang, M. Miro, and S. D. Kolev, Talanta., 2018, 189, 220.

29. T. Velempini, and K. Pillay, J. Environ. Chem. Eng., 2019, 7, 103350.

30. E. Ziaei, A. Mehdinia, and A. Jabbari, Anal. Chim. Acta., 2014, 850, 49.

31. D. Y. Qin, F. Gao, Z. H. Zhang, L. Q. Zhao, J. X. Liu, J. P. Ye, J. W. Li, and F. X. Zheng, Spectrochim. Acta B., 2013, 88, 10.

32. Z. H. Wang, Y. G. Yin, B. He, J. B. Shi, J. F. Liu, and G. B. Jiang, J. Anal. Atom. Spectrom., 2010, 25, 810.

33. Y. H. Lee, and J. Mowrer, Anal. Chim. Acta., 1989, 221, 259.

34. Y. M. Liu, F. P. Zhang, B. Y. Jiao, J. Y. Rao, and G. Leng, J. Chromatogr. A., 2017, 1493, 1.

35. R. X. Zhang, M. T. Peng, C. B. Zheng, K. L. Xu, and X. D. Hou, Microchem. J., 2016, 127, 62 .

36. X. Y. Jia, J. Y. Zhao, H. Y. Ren, J. N. Wang, Z. X. Hong, and X. Zhang, Talanta., 2019, 196, 592.

37. D. Y. Zhang, S. W. Yang, H. Y. Cheng, Y. C. Wang, and J. H. Liu, Talanta., 2019, 199, 620.

38. GB/T 5750.6-2006, standard examination methods for drinking water- metal parameters, national health commission of the people's republic of China, 2006.

39. GB 5009.17-2014, national food safety standard determination of total mercury and organic-mercury in foods, national health commission of the people's republic of China, 2014. 
Table 1 The linear regression equations, correlation coefficients and detection limits for the mercury species

\begin{tabular}{ccccc}
\hline & Linearity range & & Correlation & \\
Analytes & $\left(\mu \mathrm{g} \mathrm{L}^{-1}\right)$ & Calibration curves & coefficient, \\
& $0.20-10.0$ & $y=6.75 \times 10^{4} x+3.45 \times 10^{3}$ & 0.9991 & 0.05 \\
& $0.20-10.0$ & $y=5.15 \times 10^{4} x+5.28 \times 10^{2}$ & 0.9992 & 0.05 \\
$\mathrm{Hg}^{2+}$ & $0.40-10.0$ & $y=2.85 \times 10^{4} x+2.75 \times 10^{3}$ & 0.9996 & 0.1 \\
$\mathrm{MeHg}$ & $0.40-10.0$ & $y=3.58 \times 10^{4} x+1.03 \times 10^{4}$ & 0.9997 & 0.1 \\
$\mathrm{EtHg}$ & $\mathrm{PhHg}$ & & &
\end{tabular}

Note: LOD: limit of detection 
Table 2 The recovery yields and relative standard deviations of the mercury species

\begin{tabular}{|c|c|c|c|c|c|c|c|c|}
\hline \multirow[b]{2}{*}{ Analytes } & \multirow{2}{*}{$\begin{array}{l}\text { Background } \\
(\mu \mathrm{g} / \mathrm{L})\end{array}$} & \multirow{2}{*}{$\begin{array}{l}\text { Spiked } \\
\text { value } \\
(\mu \mathrm{g} / \mathrm{L})\end{array}$} & \multicolumn{2}{|c|}{ Lake water } & \multicolumn{2}{|c|}{ Tap water } & \multicolumn{2}{|c|}{ River water } \\
\hline & & & $\begin{array}{c}\text { Recovery } \\
(\%)\end{array}$ & $\begin{array}{l}\mathrm{RSD} \\
(\%)\end{array}$ & $\begin{array}{c}\text { Recovery } \\
(\%)\end{array}$ & $\begin{array}{l}\text { RSD } \\
(\%)\end{array}$ & $\begin{array}{c}\text { Recovery } \\
(\%)\end{array}$ & $\begin{array}{l}\text { RSD } \\
(\%)\end{array}$ \\
\hline \multirow{3}{*}{$\mathrm{Hg}^{2+}$} & \multirow{3}{*}{ N.D. } & 0.0050 & 98.0 & 4.1 & 99.2 & 4.8 & 111 & 4.3 \\
\hline & & 0.010 & 103 & 3.1 & 99.9 & 3.1 & 104 & 2.1 \\
\hline & & 0.020 & 101 & 3.5 & 112 & 4.6 & 106 & 3.4 \\
\hline \multirow{3}{*}{$\mathrm{MeHg}$} & \multirow{3}{*}{ N.D. } & 0.0050 & 99.0 & 5.1 & 109 & 2.8 & 107 & 3.1 \\
\hline & & 0.010 & 98.4 & 4.3 & 102 & 4.3 & 97.0 & 4.5 \\
\hline & & 0.020 & 97.5 & 2.9 & 99.5 & 4.3 & 104 & 4.1 \\
\hline \multirow{3}{*}{ EtHg } & \multirow{3}{*}{ N.D. } & 0.010 & 96.0 & 4.6 & 101 & 2.6 & 102 & 3.6 \\
\hline & & 0.020 & 99.4 & 4.9 & 99.0 & 3.0 & 99.2 & 4.6 \\
\hline & & 0.040 & 102 & 3.5 & 97.5 & 2.3 & 89.5 & 2.9 \\
\hline \multirow{3}{*}{$\mathrm{PhHg}$} & \multirow{3}{*}{ N.D. } & 0.010 & 93.0 & 4.6 & 90.6 & 1.7 & 87.2 & 3.2 \\
\hline & & 0.020 & 86.1 & 4.4 & 86.5 & 1.9 & 89.7 & 3.6 \\
\hline & & 0.040 & 84.0 & 4.9 & 85.9 & 1.1 & 93.3 & 6.5 \\
\hline
\end{tabular}


Table 3 Comparison of the proposed method with other methods for the determination of $\mathrm{Hg}$ species in different samples

\begin{tabular}{ccccc}
\hline samples & Hg species & $\begin{array}{c}\text { HPLC-ICP-MS } \\
\left(\mu \mathrm{g} \mathrm{L}^{-1}\right)\end{array}$ & This work $\left(\mu \mathrm{g} \mathrm{L}^{-1}\right)$ & $T$-test \\
\hline \multirow{2}{*}{ Spiked sample 1 } & $\mathrm{MeHg}$ & 0.0122 & 0.0127 & 0.76 \\
& $\mathrm{EtHg}$ & 0.0212 & 0.0198 & 0.45 \\
Spiked sample 2 & $\mathrm{MeHg}$ & 0.0210 & 0.0202 & 0.61 \\
& $\mathrm{EtHg}$ & 0.0412 & 0.0398 & 0.22 \\
River water & $\mathrm{Hg}^{2+}$ & 0.820 & 0.787 & 0.085 \\
\hline
\end{tabular}




\section{Figure Captions}

Fig. 1 The chemical structural formulas of $\mathrm{MeHg}, \mathrm{EtHg}$ and $\mathrm{PhHg}$.

Fig. 2 The effect of the elution mode and L-cysteine concentration on the chromatograms.

Fig. 3 The effect of different cartridges on the recoveries of mercury species.

Fig. 4 The effect of hydrochloric acid concentration (a) and thiourea concentration in acid solution (b) on the mercury species recoveries.

Fig. 5 The eluent volume on the mercury species recoveries. 


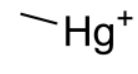

$\mathrm{MeHg}$

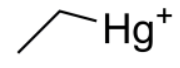

$\mathrm{EtHg}$

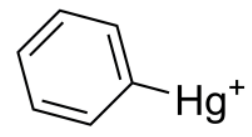

$\mathrm{PhHg}$

Fig. 1 The chemical structural formulas of $\mathrm{MeHg}$, EtHg and PhHg. 


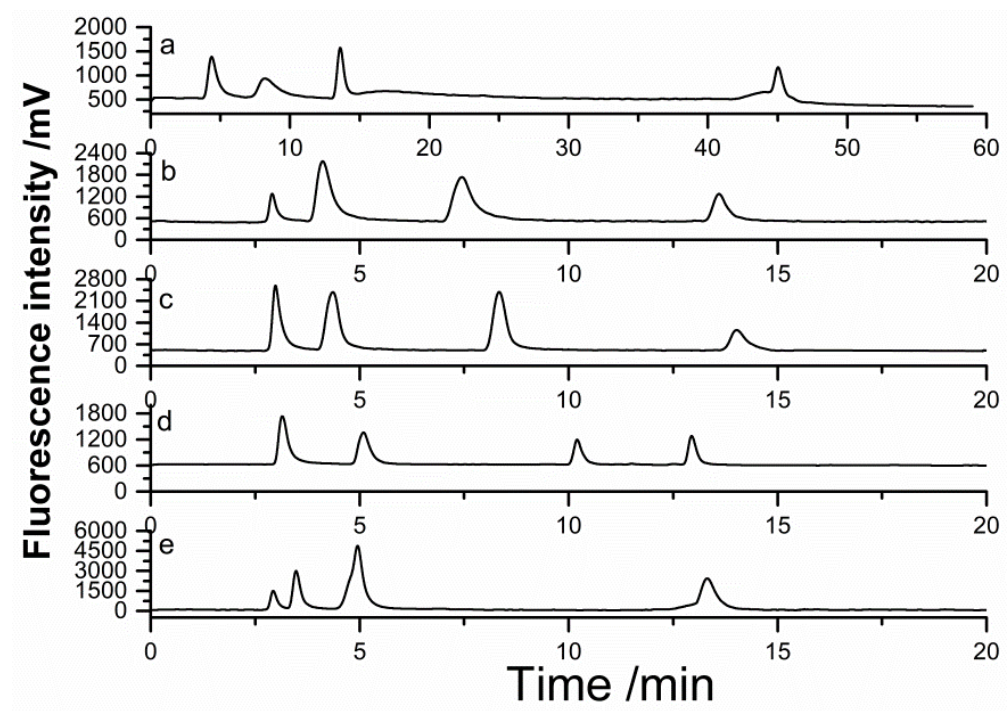

Note: a: isocratic condition without L-cysteine, b: gradient condition with $2 \mathrm{mmol} \mathrm{L}{ }^{-1}$ L-cysteine, c: gradient condition with $5 \mathrm{mmol} \mathrm{L}^{-1} \mathrm{~L}$-cysteine, d: gradient condition with $10 \mathrm{mmol} \mathrm{L}^{-1} \mathrm{~L}$-cysteine, e: gradient condition with 20 mmol L-1 L-cysteine.

Fig. 2 The effect of the elution mode and L-cysteine concentration on the chromatograms. 


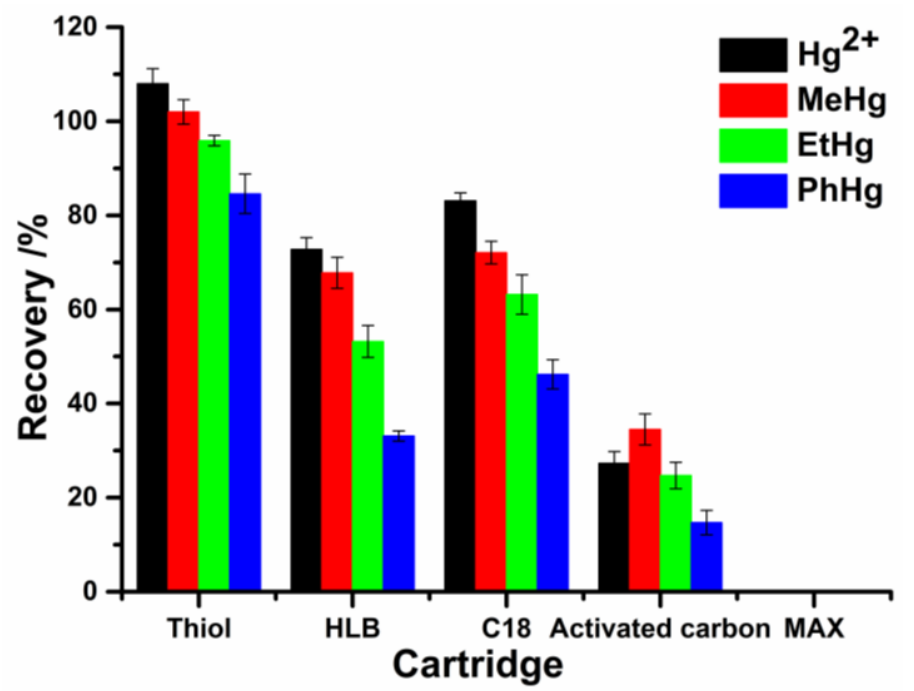

Fig. 3 The effect of different cartridges on the recoveries of mercury species. 

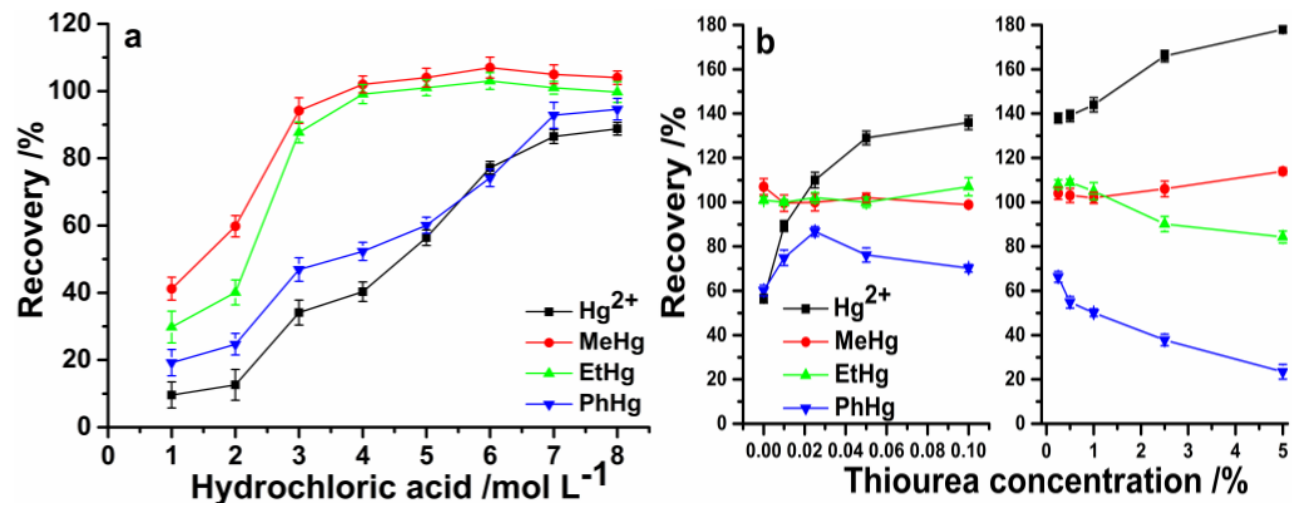

Fig. 4 The effect of hydrochloric acid concentration (a) and thiourea concentration in acid solution (b) on the mercury species recoveries. 


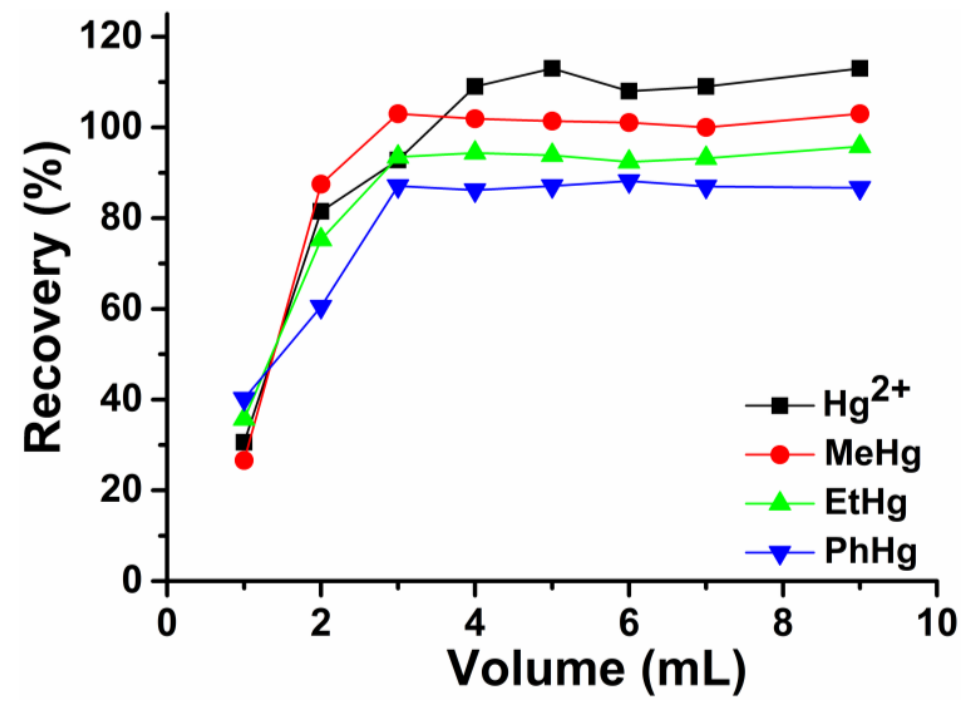

Fig. 5 The eluent volume on the mercury species recoveries. 


\section{Graphical Index}

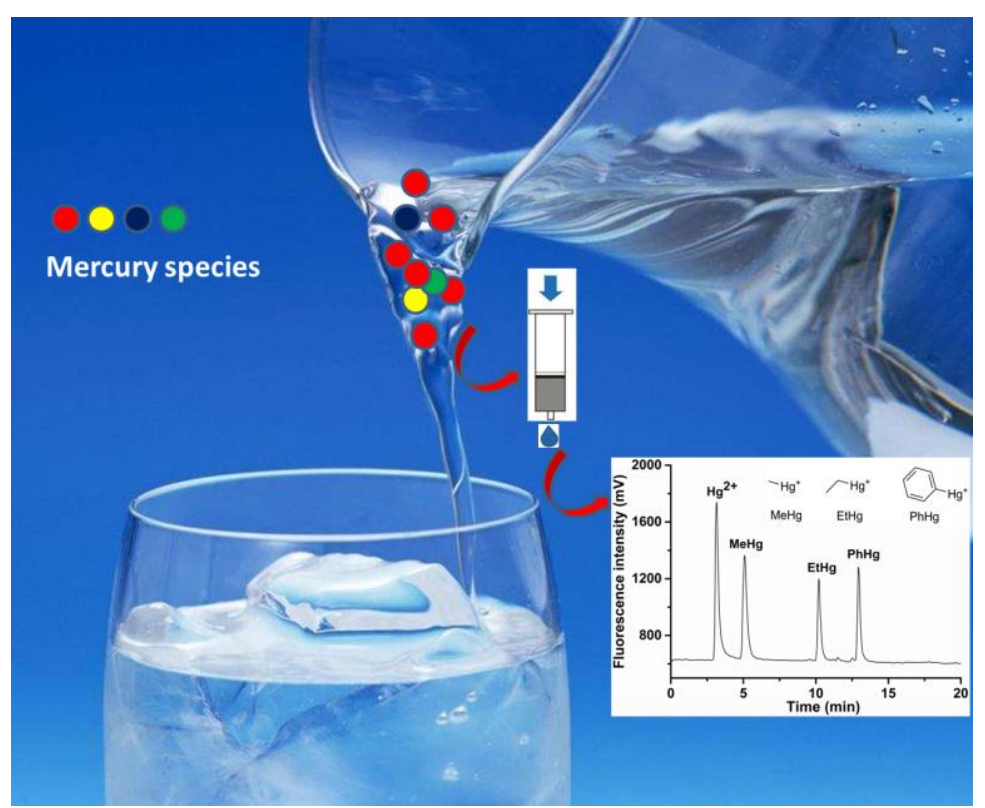

